\section{e-Journal of Educational \\ Research, Assessment and Evaluation} \section{RELIEVE}

Revista ELectrónica de Investigación y EValuación Educativa

\title{
MODELO DE COMPETENCIA DOCENTE DEL PROFESOR DE MEDICINA EN LA UNAM
}

\section{[Model of teaching competence in teachers of medicine at UNAM]}

\author{
by/por
}

$\underline{\text { Article record }}$

$\underline{\text { About authors }}$

HTML format
Flores-Hernández, Fernando (fernando.evaluacion@gmail.com)

Martínez-González, Adrián (adrianmartinez38@gmail.com)

Sánchez-Mendiola, Melchor (melchorsm@gmail.com)

García-Cabrero, Benilde (benilde@servidor.unam.mx)

Reidl, Lucy M. (lucym@servidor.unam.mx)
Ficha del artículo

$\underline{\text { Sobre los autores }}$

Formato HTML

\begin{abstract}
The study presents a model of teaching competence in teachers of medicine. It was generated from an existing conceptual model: a) it was referred to the opinion of experts who selected the competencies of greater impact; b) a reference profile was constructed; c) an instrument was generated based on student opinion, and another for selfassessment; d) the instruments were validated by 18 experts in evaluation; e) they were applied to 2,281 students and 107 teachers, obtaining adequate psychometric characteristics, establishing a model according to current trends in teacher performance assessment.
\end{abstract}

\section{Keywords}

Teachers, Assessment, Competence, Model, Performance.

\section{Resumen}

El estudio presenta un modelo de competencia docente del profesor de medicina. Fue generado a partir de un modelo conceptual preexistente: a) dicho modelo se sometió al juicio de expertos quienes seleccionaron las competencias de mayor impacto; b) se construyó un perfil de competencias; c) se generó un instrumento basado en la opinión de los estudiantes y otro de autoevaluación; d) ambos fueron validados por 18 expertos en evaluación; e) se aplicó a 2,281 estudiantes y 107 profesores, obteniendo un comportamiento psicométrico adecuado que permitió establecer un modelo acorde a las tendencias actuales en evaluación del desempeño docente.

\section{Descriptores}

Docencia, Evaluación, Competencias, Modelo, Desempeño.

de atención médica de la población. Se puede considerar que la diversidad existente proviene de fuentes como los presupuestos, programas académicos, la planta docente y el perfil de los estudiantes, entre otros aspectos, que producen rendimientos diferenciales de las escuelas (Asociación Mexicana de Facul- 
tades y Escuelas de Medicina [AMFEM], 2009).

En la actualidad no existe un censo que exprese con veracidad el número exacto de escuelas de medicina en el país, sin embargo, se estima que existen poco más de 90 instituciones que imparten la carrera en México. De éstas, alrededor de 15 se ubican en el Distrito Federal, concentrando la Universidad Nacional Autónoma de México (UNAM) a más del $50 \%$ de la demanda de primer ingreso en la entidad, considerando sus tres campus: Ciudad Universitaria (CU), Facultades de Estudios Superiores Iztacala y Zaragoza. La CU por sí misma recibe a poco más del 5\% de los estudiantes a nivel nacional (Asociación Nacional de Universidades e Instituciones de Educación Superior [ANUIES], 2007).

La Facultad de Medicina de la UNAM (2010), modificó recientemente el Plan de Estudios de la licenciatura de médico cirujano, quedando integrado como un plan por asignaturas con un enfoque por competencias, organizado en dos años de formación en ciencias básicas, dos años y medio de ciencias clínicas más un año de internado y otro de servicio social. El objetivo general del Plan es formar médicos capaces y competentes para ejercer la medicina general de cali$\mathrm{dad}$, en ambientes complejos y cambiantes mediante:

- Los conocimientos, habilidades, destrezas, actitudes y valores con ética y profesionalismo para resolver problemas de salud, integrando de manera apropiada las disciplinas biomédicas, clínicas y sociomédicas.

- La aptitud clínica para la promoción, preservación y recuperación eficaz y eficiente de la salud en individuos y poblaciones.

- El pensamiento crítico, la práctica reflexiva y la educación continua para la solución de problemas de salud, aplicando la mejor evidencia científica para la toma de decisiones clínicas.

- Las tecnologías de comunicación e información para el manejo efectivo de los problemas de salud.

Un elemento central para la consecución de los objetivos del Plan de Estudios, considerando su incidencia directa en el aprendizaje de los estudiantes, es el docente en su práctica educativa, que de acuerdo con Zabala (2002) puede ser comprendida como la actividad dinámica y reflexiva, no susceptible de ser restringida a los procesos educativos inherentes al interior del salón de clases, sino que abarca la intervención pedagógica que ocurre antes y después de la sesión de clase.

A partir del marco de referencia expuesto, un segundo elemento que juega un rol crucial para determinar la consecución de los objetivos del Plan de Estudios es la evaluación educativa. Dicha evaluación, en términos generales, pretende valorar los diversos componentes que inciden en el proceso de enseñanza aprendizaje, con el fin de poder emitir juicios sobre su mérito o valor y dar sustento a la toma de decisiones, considerando factores como la calidad, eficacia y pertinencia de diversos componentes tales como la evaluación de instituciones, planes y programas, trayectorias escolares, aprendizaje y docencia. La evaluación docente que se realiza en diversas instituciones de educación superior, valora el desempeño, habilidades, uso de recursos psicopedagógicos, estrategias de aprendizaje y apego a políticas institucionales, entre otros.

A partir de la revisión de distintos autores se puede observar que el concepto de evaluación ha tomado diversos matices (Castillo, 2002; Nevo, 1997; Joint Committee, 1981; Nirenberg, Brawerman y Ruiz, 2000; Ruiz, 1998; Scriven 1967 citado en Worthen, Sanders y Fizpatrick, 1997; Stufflebeam, 1987 y Tyler, 1977):

- Contrastar resultados contra objetivos. 
- Identificar y aplicar criterios para determinar el valor o mérito de un objeto evaluado.

- Incorporar la emisión de juicios de valor sobre el objeto evaluado.

- Responder a las necesidades de las audiencias, considerando el valor de todos los agentes involucrados.

En función de dichas definiciones y para fines del presente trabajo, la evaluación es considerada como el proceso sistemático que permite la integración cualitativa y cuantitativa de información, para determinar el valor o mérito de un objeto, donde a partir de la información recopilada se emiten juicios que orientan la toma de decisiones con fines de mejora.

Al enfocarse de manera directa en la evaluación del desempeño docente, puede definirse éste, en un sentido amplio, como la situación desarrollada en el contexto educativo a partir de la interacción que se establece entre profesores y estudiantes en el ámbito escolar, la cual se rige por una serie de reglas explícitas e implícitas determinadas por la institución y los individuos en interacción (Rueda y Torquemada, 2004).

La definición anterior se puede complementar al considerar a la docencia como una disciplina profesional, especializada y sistemática que requiere de una alta responsabilidad para su desempeño, al ser susceptible de adecuarse a las variantes particulares de cada campo disciplinar y al contexto psicopedagógico en el cual se desarrolla el proceso de enseñanza aprendizaje, involucrando diversas competencias para su ejercicio. La evaluación de los profesores en cada universidad es particular, pero puede basarse en planteamientos generales formulados previamente, sin regirse por esquemas rígidos preconcebidos ( Díaz Barriga y Ruega, 2000).

Es importante destacar que la evaluación del desempeño docente debe en todo momento ser considerada como una estrategia de mejoramiento de la educación, que entre sus funciones pretende realimentar a la realidad, delimitar diversos procedimientos y acciones ofreciendo una visión complementaria del desempeño que favorece la reflexión continua y promueve el desarrollo de sus miembros (Montenegro, 2003).

El planteamiento anterior cobra una doble relevancia al considerar la implicación de su abordaje en términos multidimensionales y multifactoriales, de acuerdo con propuestas recientes en el campo, donde se incorpora un enfoque claramente dirigido a la evaluación por competencias. De acuerdo con la Organización para la Cooperación y el Desarrollo Económico (OCDE en 2007), una competencia puede ser entendida como la capacidad para dominar un área determinada, se desarrolla en un campo particular, al aprender a tratar con tareas bien definidas y brindando respuestas efectivas para la resolución de problemas en su ámbito profesional, que son aceptadas por diversos países a nivel internacional. En este sentido, algunos de los modelos de docencia desarrollados en los últimos años que merecen una mención especial al proponer como un eje central a las competencias, las cuales sirven de marco para el desarrollo y propuesta de distintos procesos de evaluación, son: Fernández (2008), García, Loredo, Luna y Rueda (2008), Montenegro (2003), Perrenoud (2007) y Zabalza (2007).

En el contexto de la licenciatura en medicina, existen algunas propuestas que definen las competencias del médico, como las señaladas en el marco del proyecto Alfa Tuning para América Latina (2010) o las definidas desde la perspectiva profesional en el modelo del médico competente propuesto por CanMEDS, que integra seis dimensiones: profesional, comunicación, colaborador, administración, promoción de la salud y formación continua, (Royal College of Physicians and Surgeons of Canada [CANMEDS], 2006). 
Si bien la evaluación de los profesores en cada Institución de Educación Superior (IES) puede adoptar un matiz particular, un elemento común es que más allá del perfil profesional de los docentes en un campo de conocimiento, el desempeño docente implica una formación profesional, especializada y sistemática que requiere de una alta responsabilidad para su desempeño, que sea susceptible de adecuarse a las variantes particulares de cada campo disciplinar y al contexto psicopedagógico en el cual se desarrolla el proceso de enseñanza aprendizaje, involucrando diversas competencias para su ejercicio, con el objeto de facilitar a los estudiantes el perfil de competencias del egresado.

Es aquí donde la evaluación docente es primordial, relevante y de alto impacto para las IES, al constituir un elemento fundamental que brinda la posibilidad de valorar el desempeño de su planta docente y realimentar y establecer procesos de formación y actualización docente adecuados a cada institución.

Desde esta perspectiva, los procesos de evaluación desarrollados en la Facultad de Medicina de la UNAM-evaluación del aprendizaje, de planes y programas de estudio, institucional y del desempeño docenteson sustancialmente relevantes. Respecto de la evaluación del desempeño docente, desde el inicio del Plan Único de Estudios en la carrera de medicina, se mencionan a continuación los principales procesos de evaluación en la entidad que se han venido desarrollando desde 1994. Un primer instrumento de evaluación que se elaboró, denominado METEBQ-B, estaba constituido por 30 ítems, cada uno con cinco opciones de respuesta tipo Likert, que evaluaban tres dimensiones: las estrategias de enseñanza exhibidas por el docente, el respeto mostrado hacia sus alum- nos y la forma de evaluar el aprendizaje (Valle, Alaminos, Contreras, Salas, Tomasini y Varela, 2004).

Posteriormente, en 2006, se inició la aplicación de un segundo instrumento, denominado COED, que incrementó a ocho el número de dimensiones empleadas: puntualidad y asistencia, cumplimiento del programa académico, metodología pedagógica del docente, empleo de materiales y actividades de apoyo, actitud hacia los estudiantes, forma en que son evaluados los aprendizajes, actividades prácticas o de aplicación y satisfacción general de los estudiantes. El instrumento también empleaba una escala Likert y su confiabilidad global era de $\alpha=0.98$ en su conjunto (Mazón, Martínez y Martínez, 2009). Es importante destacar que, a la fecha, en la Facultad de Medicina de la UNAM sólo se aplica la evaluación basada en la opinión de los estudiantes, que brinda únicamente una visión parcial del desempeño docente, por lo que algunos autores recomiendan que esta función tan compleja, debe ser evaluada mediante varias estrategias (CANMEDS, 2006).

En 2008 un grupo de académicos de la Facultad de Medicina de la UNAM generó la propuesta de un modelo de competencias del profesor de medicina (ver Figura 1). En este modelo teórico se definieron seis competencias o dimensiones entendidas como "el conjunto de conocimientos, habilidades, actitudes y valores que posee el profesor y que relacionados entre sí permiten el desempeño exitoso de las actividades y funciones del proceso educativo". Como rasgo distintivo del modelo, las actitudes y valores se mantienen como elemento común en el contexto de la práctica docente (Martínez, López, Herrera, Ocampo, Petra, Uribe, García y Morales, 2008). 


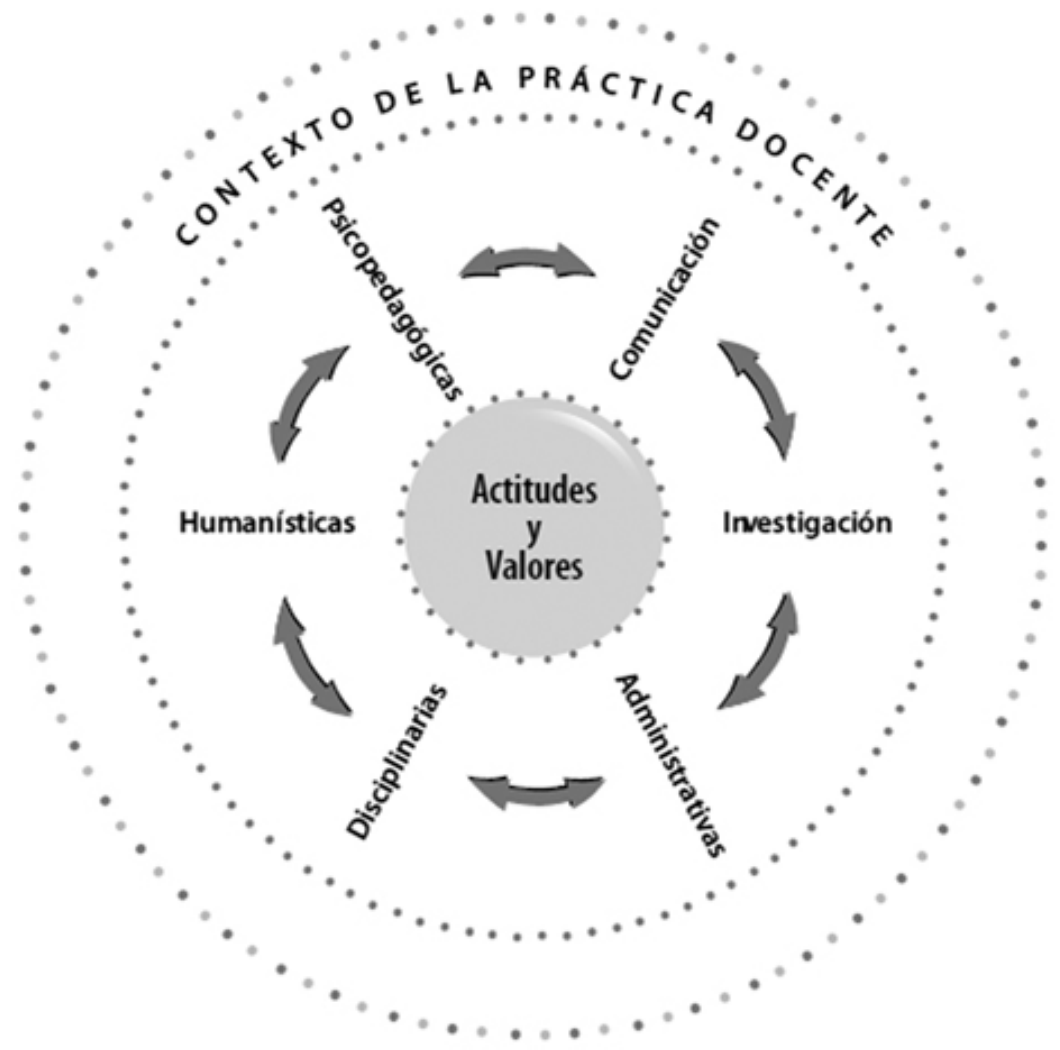

Figura 1. Modelo de competencias del profesor de medicina Tomado de (Martínez et al, 2008)

Como puede observarse en la figura 1 , se establecieron seis tipos de competencias: disciplinarias, humanísticas, psicopedagógicas, comunicación, investigación y administrativas, así como un conjunto común de actitudes y valores, que, como se señaló anteriormente, se relacionan entre sí y se ponen en operación en los diversos contextos donde se desarrolla la práctica docente del profesor de medicina. Cada una de las competencias se desglosa en el modelo mediante la descripción de una serie de actividades o funciones.

Dadas las tendencias actuales y las propuestas de diferentes autores, el presente estudio parte de una reconceptualización del modelo propuesto por Martínez, et al. (2008), en el contexto del Plan de Estudios 2010 de la Facultad de Medicina de la UNAM. Esta reconceptualización resulta congruente con el contexto de las propuestas recientes de la evaluación del desempeño docente en las IES, al llevar a la práctica el modelo propuesto a través de dos estrategias complementarias de evaluación, una basada en la opinión de los estudiantes y otra en la propia autoevaluación docente.

El presente estudio tuvo como objetivo diseñar dos instrumentos que permitieran evaluar las competencias derivadas del modelo mencionado, que se encuentra asociadas exclusivamente al desempeño docente, y con base en los resultados obtenidos realizar la propuesta inicial de un modelo de competencia docente del profesor de medicina. Y como objetivos específicos:

- Determinar las dimensiones que integran cada uno de los instrumentos propuestos.

- Contar con instrumentos de evaluación basada en la opinión de los estudiantes y autoevaluación, válidos y confiables.

- Identificar las diferencias entre las asignaturas morfológicas, fisiológicas y sociomédicas a partir de los resultados obtenidos. 


\section{Método}

El proyecto de validación del modelo se desarrolló en cinco fases:

Primera fase: las funciones y actividades definidas por Martínez et al, (2008) fueron sometidas al juicio de 74 expertos adscritos a los departamentos de las asignaturas del ciclo básico y de la Secretaría de Educación Médica de la misma Facultad, quienes seleccionaron aquellas que consideraban más asociadas al desempeño docente y las clasificaron empleando los siguientes criterios:

a) Valoraron cada función y actividad en términos de su frecuencia de uso (Nunca, Algunas veces, Frecuentemente y Siempre).

b) Ponderaron su relevancia (Nada relevante, Poco relevante, Relevante y Muy relevante).

c) Definieron si cada actividad debía ser evaluada (sí o no) y por quién (el mismo docente o los estudiantes).

Segunda fase: se seleccionaron aquellas funciones a las que el promedio de respuestas señalaban como de uso "frecuente" o "siempre" y que resultaban "relevantes" o "muy relevantes". Un primer bloque integraba las que debían ser evaluadas por los estudiantes y un segundo bloque aquellas que debían ser evaluadas por los docentes.

Tercera fase: a partir de las funciones que cumplían los criterios definidos en la etapa previa se construyó un perfil de referencia para el diseño de un instrumento de evaluación con una escala tipo Likert, basado en la opinión de los estudiantes que se denominó OPINEST2010, en el cual quedaron definidos 49 ítems asociados a las funciones seleccionadas del Modelo de Competencias del profesor de medicina.

En esta misma fase se construyó un segundo perfil que dio sustento a otro instrumento denominado OPINAUT2010, dirigido a recabar la autoevaluación docente, integrado por 52 ítems. De esta manera quedó constituida la primera versión de ambos instrumentos.

Cuarta fase: los 52 ítems se sometieron a un proceso de validación realizado por 18 expertos en evaluación educativa adscritos a la UNAM y a distintas instancias de carácter nacional vinculadas a la evaluación en las IES, con formación en el campo médico, psicológico, pedagógico, de la docencia, medición y psicometría, quienes aportaron recomendaciones en términos de la redacción y presentación de cada ítem contenido en ambos instrumentos, los cuales fueron corregidos.

Quinta fase: se realizó la aplicación piloto de 2,281 cuestionarios OPINEST2010 a una muestra de estudiantes de tres departamentos diferentes orientados a asignaturas de tipo morfológico, fisiológico y sociomédico, del primer y segundo año de la carrera de medicina. Por su parte, el cuestionario OPINAUT2010 se aplicó a una muestra de 107 profesores del ciclo básico, que aceptaron responder de manera voluntaria el instrumento.

La aplicación se desarrolló a través de los coordinadores de enseñanza de cada departamento, quienes durante alguna de las sesiones de clase solicitaban a los distintos docentes de la asignatura abandonar el aula, para aplicar el instrumento a los estudiantes de manera anónima, quienes respondían mediante hojas de lectura óptica. Respecto a los profesores, el mismo coordinador de enseñanza les entregó el cuestionario y hoja de lectura óptica para su cumplimentación y entrega.

Una vez concentrados los instrumentos y hojas de lector, se entregaron al Departamento de Evaluación de la Secretaría de Educación Médica para realizar su lectura óptica e integrar la base de datos correspondiente, para realizar el análisis psicométrico de los instrumentos. Los análisis desarrollados se 
realizaron mediante PASW 18 y AMOS 18 de SPSS Inc.

Al final del presente documento se anexa la última versión de los instrumentos empleados, actualizados a mayo de 2011 con las precisiones correspondientes derivadas de un proceso de implementación y mejora continua de la evaluación docente en la Facultad.

En cuanto a la parte ética, se realizó la invitación para participar en el estudio a los distintos departamentos del ciclo básico de la Facultad de Medicina, donde fue decisión de los mismos participar de manera libre en el estudio. De la misma forma, la respuesta a los cuestionarios fue voluntaria tanto para estudiantes como para la planta docente. En ambos casos se resguardó el anonimato de los participantes y se realimentó a los departamentos, en términos globales, sobre los resultados obtenidos.

\section{Resultados}

El estudio se desarrolló en la Facultad de Medicina de la UNAM, logrando recabar la respuesta de 2,281 estudiantes y 107 profesores de tres departamentos responsables de asignaturas con contenidos de tipo fisiológico, morfológico y sociomédico, correspondientes al ciclo básico de formación, el cual se concentra en los dos primeros años de la carrera previos al ingreso de los estudiantes a los años correspondientes al internado médico. El estudio se desarrolló entre los meses de octubre de 2010 y mayo de 2011.

En relación con el primer instrumento OPINEST2010, fue contestado por estudiantes del ciclo básico de los dos primeros años de la carrera quienes evaluaron a sus profesores. Se determinó la discriminación de dicho instrumento cortando en los percentiles .33 y .66, obteniendo un grupo inferior donde se ubicaron 757 registros, con una media de 143 \pm 18.9 puntos sobre el puntaje bruto, y un grupo superior, constituido por 776 registros, con una media de 191 \pm 5.4 . Ambos grupos se contrastaron mediante una prueba $t$ de
Student, encontrando una discriminación significativa del instrumento $[\mathrm{t}=-67.91$, $\mathrm{r}<0.01]$ asumiendo que las varianzas son iguales entre ambos grupos. Al analizar la discriminación para cada una de las 49 afirmaciones que integraron la versión original del instrumento, se encontró una discriminación significativa con una $\mathrm{r}<0.01$ para cada una de ellas. En lo concerniente a la confiabilidad del instrumento se determinó el coeficiente alfa de Cronbach, que obtuvo un valor estandarizado de $\alpha$ igual a .956 .

Posteriormente se identificó la estructura del instrumento, partiendo de un análisis factorial exploratorio de componentes principales con rotación Varimax. El empleo de la rotación ortogonal en lugar de una oblicua, se justifica porque, si bien estamos evaluando la "competencia docente" como constructo, ésta se caracteriza por diferentes dimensiones, cada una de las cuales se constituye y caracteriza por un conjunto de funciones y actividades que teóricamente se supondría que asumen independencia. Adicionalmente, en términos psicométricos, cuando las muestras son muy grandes no existe gran diferencia entre los resultados al emplear rotaciones oblicuas y ortogonales, lo cual corroboramos con nuestros datos. Algunos autores, como Ruiz y Jiménez (2004), citan diversos estudios en los cuales se han encontrado resultados muy similares comparando análisis ortogonales y oblicuos en un mismo conjunto de datos, y concluyen: "En este sentido, tal como indica Block (1995), cuando se rotan factores basados en variables que han sido previamente encajadas en categorías independientes unas de otras, no es sorprendente que las rotaciones, cuando son liberadas para ser oblicuas, encuentren poca oblicuidad y aparezcan resultados muy similares a los obtenidos por rotaciones ortogonales".

Al analizar la matriz de correlaciones sólo las afirmaciones 35,40 y 41 del instrumento presentan correlaciones no significativas con otras afirmaciones y no fueron integradas en ningún factor. Por su parte, las afirmaciones 


\section{1,2 y 6 presentan correlaciones superiores a} .9 que pueden sugerir colinealidad entre dichos reactivos. En las comunalidades, se observa que la proporción de varianza explicada para cada reactivo es superior a .40 salvo en el caso de las afirmaciones 11 (.390), 26 (.379) y 35 y 36 que no se recuperan en ningún factor como se verá más adelante.
Los valores de la matriz de correlaciones reproducida, que se obtiene a partir de la solución factorial, son un indicador de que el modelo resultante es bueno y el número de factores adecuado al reproducirse al 100\% los resultados de la extracción de cada afirmación, dando residuales igual a cero. En cuanto a la varianza explicada, sólo se toman en cuenta los seis primeros componentes o factores, que explican el 51.18\% de varianza.

Tabla 1. Competencias resultantes del OPINEST2010, obtenidas a partir de la matriz de componentes rotados y su confiabilidad

\begin{tabular}{|c|c|c|c|c|}
\hline Factor & Nombre & Afirmación & Carga & Alfa \\
\hline \multirow{19}{*}{1} & \multirow{19}{*}{$\begin{array}{l}\text { Comunicación y } \\
\text { evaluación } \\
\text { (19 } \\
\text { afirmaciones) }\end{array}$} & $\begin{array}{l}\text { Valora el avance del grupo como un referente para avanzar con los con- } \\
\text { tenidos del curso }\end{array}$ & 696 & \multirow{19}{*}{.925} \\
\hline & & $\begin{array}{l}\text { Emplea la evaluación para identificar aquellos contenidos a fortalecer en } \\
\text { el grupo, que contribuyan a mejorar el rendimiento. }\end{array}$ & 685 & \\
\hline & & $\begin{array}{l}\text { Escucha a los alumnos cuando comenta con ellos los resultados de las } \\
\text { evaluaciones. }\end{array}$ & 626 & \\
\hline & & $\begin{array}{l}\text { Establece de manera clara, los criterios que empleará para la evaluación } \\
\text { del aprendizaje. }\end{array}$ & 614 & \\
\hline & & $\begin{array}{l}\text { Emplea los resultados de la evaluación para realimentar el avance alcan- } \\
\text { zado por los alumnos. }\end{array}$ & .589 & \\
\hline & & Informa a los estudiantes sobre su desempeño en la materia. & .573 & \\
\hline & & $\begin{array}{l}\text { Da respuesta a los problemas de aprendizaje tanto individuales como de } \\
\text { grupo. }\end{array}$ & .551 & \\
\hline & & $\begin{array}{l}\text { Adecua el proceso educativo al avance del grupo, para lograr los objeti- } \\
\text { vos esperados en la asignatura. }\end{array}$ & .550 & \\
\hline & & Emplea diferentes recursos o procedimientos para evaluar el curso. & .541 & \\
\hline & & $\begin{array}{l}\text { Establece comunicación individual y grupal basándose en la compren- } \\
\text { sión apoyo y flexibilidad. }\end{array}$ & .531 & \\
\hline & & $\begin{array}{l}\text { Fomenta que el alumno autoevalúe su participación, como un medio para } \\
\text { mejorar su aprendizaje. }\end{array}$ & .510 & \\
\hline & & Propicia buena comunicación con los estudiantes. & .510 & \\
\hline & & $\begin{array}{l}\text { Fomenta una relación interpersonal en la cual los alumnos expresan de } \\
\text { manera directa y aceptable su sentir, en la emisión de juicios críticos } \\
\text { respecto a conocimientos, habilidades y actitudes propias y ajenas. }\end{array}$ & .494 & \\
\hline & & $\begin{array}{l}\text { Favorece la crítica constructiva de los alumnos frente a diversos proble- } \\
\text { mas. }\end{array}$ & .473 & \\
\hline & & $\begin{array}{l}\text { Propicia el uso de las capacidades de los propios estudiantes para interac- } \\
\text { tuar en su entorno, haciendo frente a diversas situaciones que involucran } \\
\text { la relación médico-paciente para poder superarlas. }\end{array}$ & .459 & \\
\hline & & $\begin{array}{l}\text { Analiza los principios de la relación médico-paciente en diferentes situa- } \\
\text { ciones. }\end{array}$ & .456 & \\
\hline & & $\begin{array}{l}\text { Favorece el análisis del ejercicio de la práctica médica en los sectores } \\
\text { público y privado. }\end{array}$ & .451 & \\
\hline & & $\begin{array}{l}\text { Propicia la interacción entre los alumnos para favorecer la autocrítica y } \\
\text { la crítica constructiva. }\end{array}$ & .423 & \\
\hline & & $\begin{array}{l}\text { Guía las discusiones desarrolladas en el proceso educativo, en un am- } \\
\text { biente académico de tolerancia, respeto y ecuanimidad. }\end{array}$ & .416 & \\
\hline
\end{tabular}


Flores-Hernández, Fernando; Martínez-González, Adrián; Sánchez-Mendiola, Melchor; García-Cabrero, Benilde \& Reidl, Lucy M. (2011). Modelo de competencia docente del profesor de medicina en la UNAM. RELIEVE, v. 17, n. 2, art. 3. http://www.uv.es/RELIEVE/v17n2/RELIEVEv17n2 3.htm

Tabla 1. Competencias resultantes del OPINEST2010, obtenidas a partir de la matriz de componentes rotados y su confiabilidad (continuación)

\begin{tabular}{|c|c|c|c|c|}
\hline Factor & Nombre & Afirmación & Carga & Alfa \\
\hline \multirow{6}{*}{2} & \multirow{6}{*}{$\begin{array}{l}\text { Humanística } \\
\text { (6 afirmaciones) }\end{array}$} & $\begin{array}{l}\text { Promueve la visión humanística de la profesión en el contexto de su dis- } \\
\text { ciplina. }\end{array}$ & .844 & \multirow{6}{*}{.935} \\
\hline & & $\begin{array}{l}\text { Fomenta el aprendizaje significativo de la profesión al analizar las cuali- } \\
\text { dades del médico en el ámbito profesional. }\end{array}$ & .843 & \\
\hline & & $\begin{array}{l}\text { Incluye contenidos en clase asociados a la medicina, como una profesión } \\
\text { humanística. }\end{array}$ & .837 & \\
\hline & & $\begin{array}{l}\text { Pone énfasis en la ética ligada a la práctica médica como parte del ejerci- } \\
\text { cio profesional. }\end{array}$ & .823 & \\
\hline & & $\begin{array}{l}\text { Propicia una actitud de servicio en los estudiantes como parte de la prác- } \\
\text { tica profesional del médico. }\end{array}$ & .816 & \\
\hline & & $\begin{array}{l}\text { Promueve el desarrollo de habilidades interpersonales, como parte de la } \\
\text { práctica profesional del médico. }\end{array}$ & .562 & \\
\hline \multirow{4}{*}{3} & \multirow{4}{*}{$\begin{array}{l}\text { Disciplinar } \\
\text { (4 afirmaciones) }\end{array}$} & $\begin{array}{l}\text { Relaciona en la materia diferentes hechos, condiciones y problemas aso- } \\
\text { ciados a los procesos de salud y enfermedad. }\end{array}$ & .933 & \multirow{4}{*}{960} \\
\hline & & $\begin{array}{l}\text { Aborda las características generales de la estructura y función del ser } \\
\text { humano asociadas a algunos problemas (clínicos, de salud familiar, co- } \\
\text { munitaria, etc.) de mayor frecuencia en el país. }\end{array}$ & .928 & \\
\hline & & $\begin{array}{l}\text { Generar propuestas encaminadas a la prevención, diagnóstico, tratamiento } \\
\text { y/o rehabilitación. }\end{array}$ & .917 & \\
\hline & & $\begin{array}{l}\text { Identifica problemas de salud propiciando el análisis, síntesis y evalua- } \\
\text { ción de los alumnos. }\end{array}$ & .788 & \\
\hline \multirow{6}{*}{4} & \multirow{6}{*}{$\begin{array}{l}\text { Psicopedagógico } \\
\text { (6 afirmaciones) }\end{array}$} & $\begin{array}{l}\text { Orienta al estudiante en la solución de problemas académico- } \\
\text { administrativos. }\end{array}$ & .817 & \multirow{6}{*}{.869} \\
\hline & & $\begin{array}{l}\text { Informa a los alumnos de algunos procedimientos de la administración } \\
\text { escolar en la Facultad. }\end{array}$ & .791 & \\
\hline & & $\begin{array}{l}\text { Analiza el proceso educativo, y lo ajusta empleando estrategias, (el traba- } \\
\text { jo en grupos colaborativos, la simulación, el aprendizaje basado en evi- } \\
\text { dencias, E learning) estándares y tendencias aplicables en la educación } \\
\text { médica. }\end{array}$ & .632 & \\
\hline & & Emplea diferentes estrategias de comunicación individual y de grupo. & .553 & \\
\hline & & $\begin{array}{l}\text { Favorece el reconocimiento por los estudiantes, de los logros y avances } \\
\text { de la disciplina, vinculados a problemas reales. }\end{array}$ & .449 & \\
\hline & & $\begin{array}{l}\text { Vincula los objetivos de la materia con el perfil profesional del médico } \\
\text { general. }\end{array}$ & .453 & \\
\hline \multirow{3}{*}{5} & \multirow{3}{*}{$\begin{array}{l}\text { Solución de } \\
\text { problemas } \\
\text { ( } 3 \text { afirmaciones) }\end{array}$} & $\begin{array}{l}\text { Orienta las actividades desarrolladas por los alumnos, para que propongan } \\
\text { y tomen decisiones, basados en un juicio crítico ante situaciones vincula- } \\
\text { das a los problemas de salud de mayor prevalencia. }\end{array}$ & .790 & \multirow{3}{*}{.819} \\
\hline & & $\begin{array}{l}\text { Favorece el análisis de la problemática socioeconómica, política y cultu- } \\
\text { ral a la que se enfrenta el médico en el mundo actual. }\end{array}$ & .668 & \\
\hline & & $\begin{array}{l}\text { Favorece que los estudiantes aborden, desde una perspectiva holística, los } \\
\text { diferentes problemas de salud individual o colectiva. }\end{array}$ & .571 & \\
\hline \multirow{3}{*}{6} & \multirow{3}{*}{$\begin{array}{l}\text { Intervención } \\
\text { ( } 3 \text { afirmaciones) }\end{array}$} & $\begin{array}{l}\text { Propicia en los estudiantes la toma de decisiones asociada a problemas } \\
\text { inherentes a la disciplina. }\end{array}$ & .801 & \multirow{3}{*}{.862} \\
\hline & & $\begin{array}{l}\text { Instrumenta actividades a cada situación o tema desarrollado que se vin- } \\
\text { culan a problemas planteados en la asignatura. }\end{array}$ & .800 & \\
\hline & & Muestra flexibilidad para lograr que los alumnos asuman un rol activo. & .800 & \\
\hline
\end{tabular}

Se realizó un análisis confirmatorio mediante un modelo de ecuaciones estructurales, obteniendo un valor de $\mathrm{Chi}^{2}$ de 4.37 $(r<0.01$. Los reactivos que integran cada di- mensión obtuvieron pesos de regresión estandarizados significativos, que oscilaron entre .540 y .988 . 
También cabe considerar el valor del error de aproximación cuadrático medio RMSEA, que de acuerdo con Hair (2007), corrige la tendencia del valor de $\mathrm{Chi}^{2}$ para rechazar modelos al tener una muestra grande, siendo un valor de bondad de ajuste esperada sobre la población y no sólo sobre la muestra extraída. El valor obtenido fue de .038 (se consideran aceptables los valores inferiores a .08) y el índice de ajuste normal obtenido NFI fue de .984 (su valor se asume entre 0 mal ajuste y 1 ajuste perfecto).

Con excepción de la competencia disciplinaria que tiene un comportamiento distinto (los alumnos afirman que más del 10\% de los profesores hace uso siempre de esta competencia, el $65.5 \%$ algunas veces o frecuentemente, el 19\% nunca y alrededor del 5\% consideran que no aplica para su asignatura), las competencias restantes se comportan de la siguiente manera: entre el del $70 \%$ y $80 \%$ de los estudiantes coincide en que los docentes aplican las habilidades y funciones que las integran de manera frecuente, alrededor del $20 \%$ coinciden en que son empleadas algunas veces y menos del 1\% considera que no aplican para su asignatura. La distribución de los porcentajes de respuesta asociados a cada competencia, se muestra en la Tabla 2.

Tabla 2. Distribución de porcentajes de respuesta por cada competencia obtenidos del OPINEST2010

\begin{tabular}{lccccc}
\hline \multicolumn{1}{c}{ Factor } & No aplica & Nunca & Algunas veces & Frecuentemente & Siempre \\
\hline $\begin{array}{l}\text { Comunicación y } \\
\text { evaluación }\end{array}$ & $0 \%$ & $2.8 \%$ & $20.4 \%$ & $73.6 \%$ & $3.2 \%$ \\
Humanística & $.6 \%$ & $3.8 \%$ & $13.9 \%$ & $78.6 \%$ & $3.1 \%$ \\
Disciplinaria & $5.2 \%$ & $19 \%$ & $28.4 \%$ & $37.1 \%$ & $10.3 \%$ \\
$\begin{array}{l}\text { Psicopedagógica } \\
\begin{array}{l}\text { Solución de } \\
\text { problemas }\end{array}\end{array}$ & $.1 \%$ & $3.6 \%$ & $15.6 \%$ & $76 \%$ & $4.7 \%$ \\
Aplicación & $.6 \%$ & $5.6 \%$ & $17.4 \%$ & $74.2 \%$ & $2.2 \%$ \\
\hline
\end{tabular}

De manera operacional y en función de los indicadores propuestos en los reactivos, aseveraciones o afirmaciones que evalúan cada competencia, para fines de este proyecto se propone la siguiente definición conceptual:

La primera competencia, comunicación y evaluación, está asociada a la comunicación del docente con los estudiantes, efectiva y abierta, tanto grupal como individual, para identificar sus necesidades, adecuar su planeación a las características del grupo, fomentar la expresión de ideas de manera asertiva, emisión de juicios críticos y autocrítica, relacionadas con su desempeño y el de los demás en el contexto de diversos problemas de salud. Por otra parte, se refiere también al manejo de un esquema de evaluación integral dirigido a aspectos cualitativos y cuantitativos, donde el docente considera como elementos eje: el avance del grupo, la identificación de los contenidos a fortalecer, el empleo de un proceso transparente con criterios definidos y abiertos de evaluación y el empleo de diversas estrategias de autoevaluación y evaluación que le permiten interactuar y realimentar a los alumnos para mejorar su rendimiento académico.

La segunda competencia, humanística, se caracteriza porque el docente fomenta la generación de una visión humanística y ética en el contexto de la práctica médica de la disciplina, basada en el aprendizaje significativo y el desarrollo de habilidades interpersonales.

La tercera competencia, disciplinar, establece que el docente hace alusión a la relación que se establece entre los contenidos de 
la asignatura y diversos problemas y situaciones en el contexto médico: aspectos asociados a los procesos de salud o enfermedad; a la estructura y funciones de los seres humanos, encaminados a los diferentes niveles de atención.

La cuarta competencia, psicopedagógica, se caracteriza por la comunicación docenteestudiante en dos niveles, individual y grupal, orientada a la resolución de problemas académicos, la adecuación de la planeación docente a las necesidades del grupo, la vinculación y actualización a los avances de la disciplina, las estrategias didácticas y la relación de los contenidos con los perfiles de la carrera.

La quinta competencia, solución de problemas, señala que el docente favorece el abordaje integral de diversos problemas de salud, dirigiendo las actividades de los estudiantes al análisis, juicio clínico, elaboración de propuestas de abordaje de la situación y toma de decisiones fundamentadas en la evidencia.

La sexta competencia, aplicación, se refiere a que el docente se orienta al logro de un rol activo por parte de los estudiantes por medio del planteamiento de diversos problemas inherentes a la disciplina, que favorezcan la aplicación de los contenidos en la práctica clínica para la toma de decisiones.

En relación con los tres departamentos académicos participantes, se observó que el patrón de respuesta en las medias de las seis competencias es prácticamente el mismo más/menos una o dos décimas en los valores obtenidos (Figura 2).

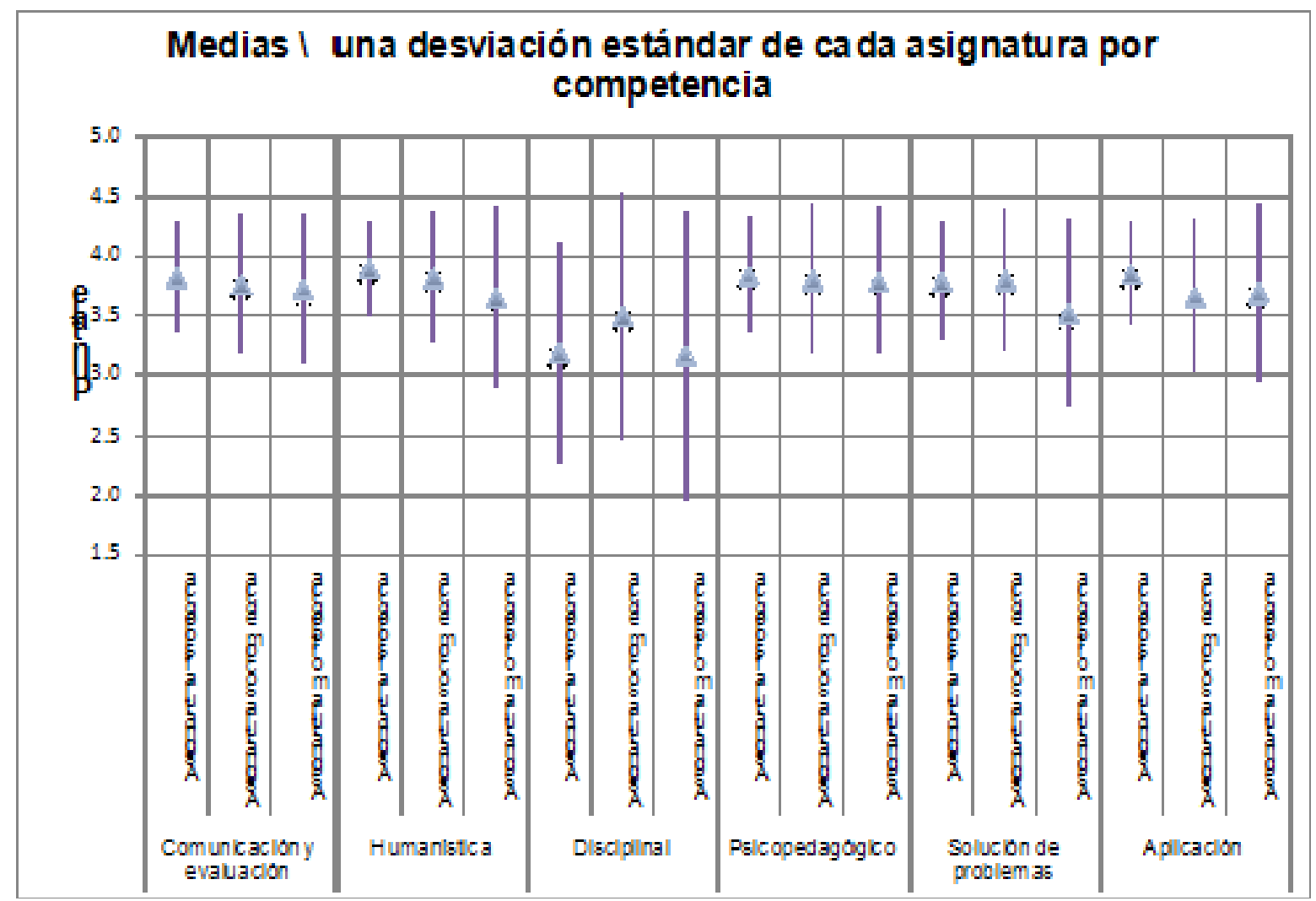

Figura 2. Medias y desviación estándar de las seis competencias según asignatura.

En las competencias de comunicación y evaluación, humanística, disciplinar, solución de problemas y aplicación se encontra- ron diferencias significativas entre las tres asignaturas $[\mathrm{r}<0.01]$ al aplicar un ANOVA, con la prueba de Tukey como post-hoc (Tabla 3). Únicamente se encontró un resultado 
no significativo en la competencia psicopedagógica, donde se observa una media igual en los tres grupos (3.8 puntos) como se observa en la Figura 2.

Tabla 3. Diferencias significativas por asignaturas mediante la prueba de Tukey HSD dl OPINEST2010

\begin{tabular}{|c|c|c|c|c|c|c|c|}
\hline \multirow{3}{*}{$\begin{array}{l}\text { Variable } \\
\text { dependiente }\end{array}$} & \multicolumn{5}{|c|}{ Comparación múltiple } & & \\
\hline & \multirow[t]{2}{*}{ (I) Asignatura } & \multirow[t]{2}{*}{ (J) Asignatura } & \multirow{2}{*}{$\begin{array}{c}\text { Diferencia de } \\
\text { medias (I-J) }\end{array}$} & \multirow{2}{*}{$\begin{array}{c}\text { Error } \\
\text { estándar }\end{array}$} & \multirow[t]{2}{*}{ Sig. } & \multicolumn{2}{|c|}{$\begin{array}{c}95 \% \text { Intervalo de } \\
\text { confianza }\end{array}$} \\
\hline & & & & & & Límite bajo & Límite alto \\
\hline \multirow{4}{*}{$\begin{array}{l}\text { Comunicación } \\
\text { y evaluación } \\
\text { Humanística }\end{array}$} & fisiológica & morfológica & 0.101 & .028 & .001 & .034 & .168 \\
\hline & fisiológica & sociomédica & 0.076 & .028 & .019 & .010 & .142 \\
\hline & & morfológica & 0.245 & .030 & .000 & .176 & .315 \\
\hline & sociomédica & morfológica & 0.170 & .030 & .000 & .098 & .241 \\
\hline \multirow[t]{2}{*}{ Disciplinar } & fisiológica & sociomédica & -0.317 & .051 & .000 & -.438 & -.197 \\
\hline & sociomédica & morfológica & 0.332 & .055 & .000 & .202 & .462 \\
\hline \multirow{2}{*}{$\begin{array}{l}\text { Solución de } \\
\text { problemas }\end{array}$} & fisiológica & morfológica & 0.267 & .032 & .000 & .192 & .343 \\
\hline & sociomédica & morfológica & 0.272 & .033 & .000 & .195 & .349 \\
\hline \multirow[t]{2}{*}{ Aplicación } & fisiológica & sociomédica & 0.184 & .030 & .000 & .114 & .254 \\
\hline & & morfológica & 0.168 & .031 & .000 & .095 & .242 \\
\hline
\end{tabular}

La diferencia de medias es significativa a un nivel de 0.05 para los datos presentados

En lo concerniente al segundo instrumento OPINAUT2010, que fue contestado por 107 profesores, en primera instancia se determinó la discriminación, obteniendo un grupo inferior donde se ubicaron 35 profesores con una media de $67.02 \pm 7.49$ puntos sobre el puntaje bruto y en el grupo superior 36 registros con una media de $95.52 \pm 11.84$.

Al someter al instrumento a la prueba $t$ de Student, se observa una discriminación significativa del instrumento [ $\mathrm{t}=-33.33, \mathrm{r}<0.01]$, asumiendo que las varianzas son iguales entre ambos grupos. En lo concerniente a la confiabilidad se determinó el coeficiente alfa de Cronbach, obteniendo un valor estandarizado de $\alpha$ igual a .978 .

Posteriormente se identificó la estructura del instrumento, partiendo de un análisis factorial exploratorio de componentes principales con rotación Varimax. Dado el tamaño reducido de la muestra es importante considerar algunos indicadores adicionales sobre el análisis, concretamente, el valor de Kaiser-Meyer-Olkin (KMO), que permite estimar la factibilidad de llevar a cabo un análisis factorial al comparar los valores de los coeficientes de correlación observados con los coeficientes de correlación parcial. En este caso, el valor obtenido es de .911. No existen correlaciones superiores a .9 que pudiesen sugerir una colinealidad entre los reactivos que integran el instrumento.

En las comunalidades obtenidas se obtuvieron varianzas explicadas para cada reactivo entre .601 y .851. Los valores de la matriz de correlaciones reproducida se ajustaron a las comunalidades obtenidas, como un indicador de que el modelo resultante es bueno y el número de factores adecuado al reproducir al $100 \%$ los resultados de la extracción de cada afirmación, dando residuales igual a cero. En relación con la varianza explicada, sólo se obtuvieron dos factores, que permiten explicar el $46.55 \%$ de varianza.

El instrumento quedó integrado por 40 afirmaciones, los factores o competencias resultantes se integran en la Tabla 4, donde se presentan el número de competencia, su nombre y reactivos que la integran, el rango de las cargas factoriales asociadas a los reactivos y el valor del alfa de Cronbach. 
Flores-Hernández, Fernando; Martínez-González, Adrián; Sánchez-Mendiola, Melchor; García-Cabrero, Benilde \& Reidl, Lucy M. (2011). Modelo de competencia docente del profesor de medicina en la UNAM. RELIEVE, v. 17, n. 2, art. 3. http://www.uv.es/RELIEVE/v17n2/RELIEVEv17n2 3.htm

Tabla 4. Competencias resultantes del OPINAUT2010, obtenidas a partir de la matriz de componentes rotados y su confiabilidad

\begin{tabular}{|c|c|c|c|c|c|}
\hline Factor & Nombre & No. & Afirmación & Carga & Alfa \\
\hline \multirow{21}{*}{. } & \multirow{21}{*}{$\begin{array}{l}\text { Docencia }(35 \\
\text { afirmaciones) }\end{array}$} & R16 & $\begin{array}{l}\text { Orienta las discusiones académicas con los alumnos en un am- } \\
\text { biente de tolerancia, respeto y ecuanimidad. }\end{array}$ & 0.867 & \\
\hline & & R11 & Promueve la motivación e interés del grupo & 0.863 & \\
\hline & & R39 & $\begin{array}{l}\text { Participa en los procesos asignados para su evaluación docente } \\
\text { y realiza las acciones correctivas pertinentes }\end{array}$ & 0.845 & \\
\hline & & R19 & $\begin{array}{l}\text { Estimula la búsqueda de información válida y confiable tanto en } \\
\text { inglés como en español y la integración de la misma. }\end{array}$ & 0.833 & \\
\hline & & R34 & $\begin{array}{l}\text { Considera fundamental brindar opciones que propicien el } \\
\text { aprendizaje autorregulado de los estudiantes. }\end{array}$ & 0.828 & \\
\hline & & R43 & $\begin{array}{l}\text { Planea, desarrolla y evalúa su práctica docente, ajustando sus } \\
\text { estrategias a las condiciones de aprendizaje individual y de } \\
\text { grupo en su asignatura. }\end{array}$ & 0.826 & \\
\hline & & R32 & $\begin{array}{l}\text { Promueve mediante preguntas, que el alumno identifique sus } \\
\text { necesidades y problemáticas como un recurso didáctico. }\end{array}$ & 0.809 & \\
\hline & & R40 & $\begin{array}{l}\text { Mantiene actualizado su conocimiento en la disciplina y su } \\
\text { formación docente. }\end{array}$ & 0.798 & \\
\hline & & $\mathrm{R} 12$ & $\begin{array}{l}\text { Emplea diversas estrategias y recursos didácticos dirigidos a } \\
\text { lograr las competencias de la asignatura. }\end{array}$ & 0.785 & \\
\hline & & R38 & $\begin{array}{l}\text { Actúa frente a sus alumnos y reflexiona con ellos, manteniendo } \\
\text { la imparcialidad ante ideologías, creencias y preferencias de } \\
\text { etnia, género y edad en la práctica médica. }\end{array}$ & 0.782 & \\
\hline & & R42 & $\begin{array}{l}\text { Analiza su práctica docente y reconoce fortalezas y debilidades } \\
\text { en la misma. }\end{array}$ & 0.772 & \\
\hline & & R14 & $\begin{array}{l}\text { Fomenta el uso y búsqueda de información formal, de fuentes } \\
\text { reconocidas en diferentes medios por parte de los alumnos. }\end{array}$ & 0.771 & \multirow[t]{10}{*}{0.984} \\
\hline & & $\mathrm{R} 18$ & $\begin{array}{l}\text { Propicia que los estudiantes fundamenten sus aseveraciones y } \\
\text { defiendan su postura, a partir de la exposición de juicios críticos } \\
\text { de diferentes fuentes. }\end{array}$ & 0.768 & \\
\hline & & R28 & $\begin{array}{l}\text { Modera su práctica docente en términos del manejo y caracte- } \\
\text { rísticas del grupo. }\end{array}$ & 0.755 & \\
\hline & & R36 & $\begin{array}{l}\text { Muestra flexibilidad al reflexionar con los alumnos sobre diver- } \\
\text { sos problemas y situaciones que involucran aspectos éticos. }\end{array}$ & 0.751 & \\
\hline & & R41 & $\begin{array}{l}\text { Integra las nuevas tendencias psicopedagógicas aplicables a la } \\
\text { educación médica en su actividad docente. }\end{array}$ & 0.743 & \\
\hline & & R33 & $\begin{array}{l}\text { Estimula el trabajo activo del alumno, fungiendo como asesor y } \\
\text { guía en el desarrollo del curso. }\end{array}$ & 0.743 & \\
\hline & & R31 & $\begin{array}{l}\text { Favorece el aprendizaje autoregulado de los estudiantes, me- } \\
\text { diante el trabajo en grupos colaborativos y el uso de recursos } \\
\text { psicopedagógicos }\end{array}$ & 0.742 & \\
\hline & & R13 & $\begin{array}{l}\text { Valora su práctica docente, las características propias de cada } \\
\text { grupo y las competencias logradas por el estudiante, como ele- } \\
\text { mento de mejora. }\end{array}$ & 0.715 & \\
\hline & & R35 & $\begin{array}{l}\text { Propicia la actitud ética y reflexiva de los estudiantes ante situa- } \\
\text { ciones que brinden elementos, para mejorar el manejo de sus } \\
\text { emociones en su ejercicio profesional. }\end{array}$ & 0.703 & \\
\hline & & R37 & $\begin{array}{l}\text { Analiza diferentes situaciones de relación médico-paciente que } \\
\text { involucran la visión humanista y ética, en su práctica profesio- } \\
\text { nal. }\end{array}$ & 0.674 & \\
\hline
\end{tabular}


Flores-Hernández, Fernando; Martínez-González, Adrián; Sánchez-Mendiola, Melchor; García-Cabrero, Benilde \& Reidl, Lucy M. (2011). Modelo de competencia docente del profesor de medicina en la UNAM. RELIEVE, v. 17, n. 2, art. 3. http://www.uv.es/RELIEVE/v17n2/RELIEVEv17n2 3.htm

Tabla 4. Competencias resultantes del OPINAUT2010, obtenidas a partir de la matriz de componentes rotados y su confiabilidad (continuación)

\begin{tabular}{|c|c|c|c|c|c|}
\hline Factor & Nombre & No. & Afirmación & Carga & Alfa \\
\hline \multirow{14}{*}{1} & \multirow{14}{*}{$\begin{array}{l}\text { Docencia }(35 \\
\text { afirmaciones) }\end{array}$} & $\mathrm{R} 17$ & $\begin{array}{l}\text { Cuando establece una problemática determinada, fomenta que } \\
\text { los alumnos tomen decisiones basadas en un juicio crítico. }\end{array}$ & 0.667 & \multirow{14}{*}{0.984} \\
\hline & & $\mathrm{R} 48$ & $\begin{array}{l}\text { Tiene presentes las competencias inherentes al perfil profesio- } \\
\text { nal de la carrera de medicina. }\end{array}$ & 0.666 & \\
\hline & & $\mathrm{R} 10$ & $\begin{array}{l}\text { Promueve el autoconocimiento del alumno, adecuándose a sus } \\
\text { estilos de aprendizaje. }\end{array}$ & 0.660 & \\
\hline & & R7 & $\begin{array}{l}\text { Emplea instrumentos y métodos de evaluación, que permitan } \\
\text { medir el nivel de logro de los estudiantes en relación con las } \\
\text { actividades propuestas en el curso. }\end{array}$ & 0.657 & \\
\hline & & $\mathrm{R} 45$ & $\begin{array}{l}\text { Maneja diversas estrategias de comunicación que le permiten } \\
\text { interactuar con los estudiantes, pares, y/o pacientes, etc. }\end{array}$ & 0.649 & \\
\hline & & R24 & Favorece la reflexión sobre la realidad actual del ámbito médico & 0.644 & \\
\hline & & R27 & $\begin{array}{l}\text { Estimula la curiosidad científica poniendo énfasis en las venta- } \\
\text { jas del conocimiento inter y transdisciplinario, en el contexto } \\
\text { nacional e internacional. }\end{array}$ & 0.643 & \\
\hline & & R15 & $\begin{array}{l}\text { Favorece que el alumno exprese su juicio crítico con respecto a } \\
\text { conocimientos, habilidades y actitudes, tanto propias como } \\
\text { ajenas, de manera asertiva y constructiva. }\end{array}$ & 0.618 & \\
\hline & & R23 & $\begin{array}{l}\text { Adecua las sesiones de clase a lo largo del curso, incorporando } \\
\text { recursos psicopedagógicos que estimulan el conocimiento de la } \\
\text { disciplina. }\end{array}$ & 0.605 & \\
\hline & & R25 & $\begin{array}{l}\text { Destaca para los alumnos el planteamiento de ciertos problemas } \\
\text { médicos de acuerdo a su nivel académico y contexto de la pro- } \\
\text { fesión. }\end{array}$ & 0.575 & \\
\hline & & R9 & $\begin{array}{l}\text { Valora de manera cualitativa las competencias mostradas por } \\
\text { los alumnos, como un elemento del proceso formativo. }\end{array}$ & 0.561 & \\
\hline & & R30 & $\begin{array}{l}\text { Promueve la participación en la enseñanza, difusión del cono- } \\
\text { cimiento y en el manejo de fuentes de información científica. }\end{array}$ & 0.539 & \\
\hline & & R49 & $\begin{array}{l}\text { Mantiene actualizado su conocimiento del contexto institucional } \\
\text { universitario de la carrera de medicina. }\end{array}$ & 0.538 & \\
\hline & & R20 & $\begin{array}{l}\text { Propicia el uso del método científico, clínico y/o epidemiológi- } \\
\text { co, como fundamento para buscar e interpretar información } \\
\text { sobre problemas médicos. }\end{array}$ & 0.496 & \\
\hline Factor & Nombre & No. & Afirmación & Carga & Alfa \\
\hline \multirow{5}{*}{2} & \multirow{5}{*}{$\begin{array}{l}\text { Solución de } \\
\text { problemas }(5 \\
\text { afirmaciones })\end{array}$} & R5 & $\begin{array}{l}\text { Destaca la recopilación y ordenamiento de diferentes resultados } \\
\text { clínicos y epidemiológicos, para plantear acciones encaminadas } \\
\text { a la solución de diferentes problemas de salud. }\end{array}$ & 0.795 & \multirow{5}{*}{0.847} \\
\hline & & $\mathrm{R} 1$ & $\begin{array}{l}\text { Guía a los estudiantes en la identificación y solución de pro- } \\
\text { blemas asociados a las enfermedades de mayor trascendencia y } \\
\text { relevancia en el país. }\end{array}$ & 0.782 & \\
\hline & & R4 & $\begin{array}{l}\text { Establece una visión holística de la medicina que favorezca la } \\
\text { selección de estrategias y acciones encaminadas a abordar los } \\
\text { problemas de salud de alta trascendencia y prevalencia. }\end{array}$ & 0.743 & \\
\hline & & R3 & $\begin{array}{l}\text { Plantea problemas de salud, promoviendo en los estudiantes el } \\
\text { uso del análisis, síntesis y evaluación, para la implementación } \\
\text { de diversas estrategias y acciones de prevención, diagnóstico, } \\
\text { tratamiento y/o rehabilitación. }\end{array}$ & 0.718 & \\
\hline & & R2 & $\begin{array}{l}\text { Relaciona las características y funciones del ser humano, como } \\
\text { un elemento relevante para proponer soluciones a diferentes } \\
\text { problemas de salud. }\end{array}$ & 0.647 & \\
\hline
\end{tabular}


La distribución de los porcentajes de respuesta asociados a cada competencia, se muestra en la Tabla 5, donde se observa que el $63.5 \%$ de los docentes afirma que hace uso de la competencia de habilidades docentes de manera frecuente o siempre mientras que el $54.2 \%$ lo hace con la competencia de solución de problemas. El $20.6 \%$ y $14 \%$, respectivamente, afirman que nunca hacen uso de dichas competencias.

Tabla 5. Distribución de porcentajes de respuesta por cada competencia obtenidos del OPINAUT2010

\begin{tabular}{lcccc}
\hline Factor & Nunca & Algunas veces & Frecuentemente & Siempre \\
\hline Habilidades docentes & $20.6 \%$ & $15.9 \%$ & $59.8 \%$ & $3.7 \%$ \\
Solución de problemas & $14 \%$ & $31.8 \%$ & $43.9 \%$ & $10.3 \%$ \\
\hline
\end{tabular}

De manera operacional ambas competencias se definen de la siguiente manera. La competencia de Habilidades docentes, está asociada a mantener una adecuada comunicación docente-estudiante, individual y grupal, orientar las discusiones grupales, motivar y mantener el interés del grupo, fomentar el juicio crítico y la toma de decisiones de los estudiantes, participar y desarrollar diversos procesos de evaluación, orientar la búsqueda de información válida y confiable, propiciar el aprendizaje auto regulado y significativo tanto a nivel individual como de grupo, realizar un uso adecuado de los recursos y estrategias didácticas, autoevaluar su desempeño docente, mantener y fomentar un ambiente ético de tolerancia y respeto, brindar asesoría a los estudiantes y fomentar el interés por la aplicación de la metodología de investigación.

La segunda competencia asociada a la solución de problemas se caracteriza por la asesoría a los estudiantes para la recopilación y ordenamiento de diferentes resultados clínicos y epidemiológicos, la generación de una visión holística que favorezca la selección de estrategias y acciones encaminadas a abordar los problemas de salud, la promoción del análisis, síntesis y evaluación, para la implementación de diversas estrategias y acciones de prevención, diagnóstico, tratamiento y/o rehabilitación.

Se realizó un análisis confirmatorio mediante un modelo de ecuaciones estructurales obteniendo un valor de $\mathrm{Chi}^{2}$ de 26.71 $(\mathrm{r}<0.01)$ y un RMSEA de .22. Aunque son datos indicativos de una ausencia de ajuste, el índice NFI obtenido, sin embargo, fue de .898 .

\section{Discusión}

Este trabajo tomó como referente el Modelo de Competencias del Profesor de Medicina de la Facultad y se inició con la selección de las funciones y actividades concernientes exclusivamente al ejercicio docente por parte de expertos en el ámbito médico, quienes definieron qué funciones podía evaluar la planta docente y cuáles los estudiantes. A partir del proceso de construcción de los instrumentos, validación por expertos en evaluación pertenecientes a distintas instancias y su análisis psicométrico, se presenta la siguiente discusión en torno a los resultados obtenidos en el presente estudio.

En la revisión de la literatura se pueden observar diversos modelos y propuestas que abordan exclusivamente las competencias docentes como los propuestos por (Perrenoud, 2007; Zabalza, 2007; Fernández, 2008; García et al, 2008) y otros que se enfocan exclusivamente a las competencias clínicas y profesionales del médico, como son las propuestas del proyecto Tuning 2010 y el modelo del Royal College of Physicians and Surgeons of Canada (2006). Lo anterior se confirma al considerar una revisión sistemática de la literatura en la cual se valora el contenido y calidad de diversos cuestionarios empleados para la evaluación de profesores clínicos, que se encuentran orientados a identificar los objetivos, fortalezas y debilidades de los profesores. Dicha revisión se realizó 
en diversas bases de datos, como MEDLINE, EMBASE, PsycINFO y ERIC, desde 1976 hasta marzo de 2010 y reporta 54 trabajos basados en 32 instrumentos distintos, donde se encontraron referentes asociados a su contenido, como estrategias de enseñanza, roles, opinión, actividades de aprendizaje, buenas prácticas en el contexto clínico y algunos datos psicométricos de los mismos (Fluit, Bolhuis, Grol, Laan y Wensing, 2010).

En relación con el modelo planteado por Martínez et al. (2008), se puede considerar fundamental su aporte como un precedente y fundamento del presente estudio, ya que implicó un avance sustancial el contar con una definición previa de las funciones y actividades involucradas en el desempeño docente de los profesores, definidas ex profeso y en el contexto propio de la Facultad de Medicina de la UNAM. Esta definición permitió generar los instrumentos OPINEST2010 y OPINAUT2010 para los procesos de evaluación docente de la misma entidad en el marco de la implementación del Plan de Estudios 2010.

Los resultados obtenidos se pueden considerar relevantes a corto y medio plazo, ya que posibilitan la realización de un estudio institucional que amplíe el alcance de la evaluación a los docentes de la fase clínica, de manera complementaria a las asignaturas del ciclo básico (biomédicas y sociomédicas) representadas por los tres departamentos participantes, con el fin de hacer extensivo el modelo a todos los profesores de la facultad.

Derivado del desarrollo y resultados obtenidos en el presente estudio, se puede concluir en relación con el objetivo planteado que ambos instrumentos cubren los criterios necesarios de confiabilidad y validez para su aplicación, además de permitir discriminar entre los docentes de alto y bajo desempeño a partir de los puntajes obtenidos analizados mediante la prueba $t$ de Student. Respecto al instrumento OPINAUT2010, es importante destacar que pese a que se obtuvo un valor elevado de $\mathrm{Chi}^{2}$ y RMSEA en el análisis confirmatorio mediante modelos de ecuaciones estructurales, esto puede ser un factor atribuible al tamaño de la muestra. Por ello se considera pertinente tomar dichos valores con reserva para su análisis posterior con una muestra mayor.

Es indispensable continuar con esta línea de investigación para valorar la consistencia y generalizabilidad del instrumento en el marco de otras facultades y escuelas de medicina tanto nacionales como del extranjero, partiendo del hecho de que las competencias docentes y médicas propias de diversos programas de pregrado mantienen una base común, definidas incluso en el contexto clínico por instancias como el proyecto Tuning $\mathrm{y}$ Alfa Tuning para Europa y América Latina respectivamente.

Por otra parte, los resultados obtenidos permiten sustentar una propuesta, derivada del modelo original de competencias del profesor de medicina que incluía todas las actividades y funciones de los profesores en la entidad. En esta nueva propuesta de un Modelo de Competencias Docentes del Profesor de Medicina se mantienen las dimensiones disciplinar, psicopedagógica y humanística pero orientadas exclusivamente a la práctica docente y se amplían las dimensiones asociadas a la comunicación y evaluación, la solución de problemas y la aplicación. Estas últimas reemplazan a las propuestas originalmente de investigación y administrativa, tal como se muestra en la Figura 3. 


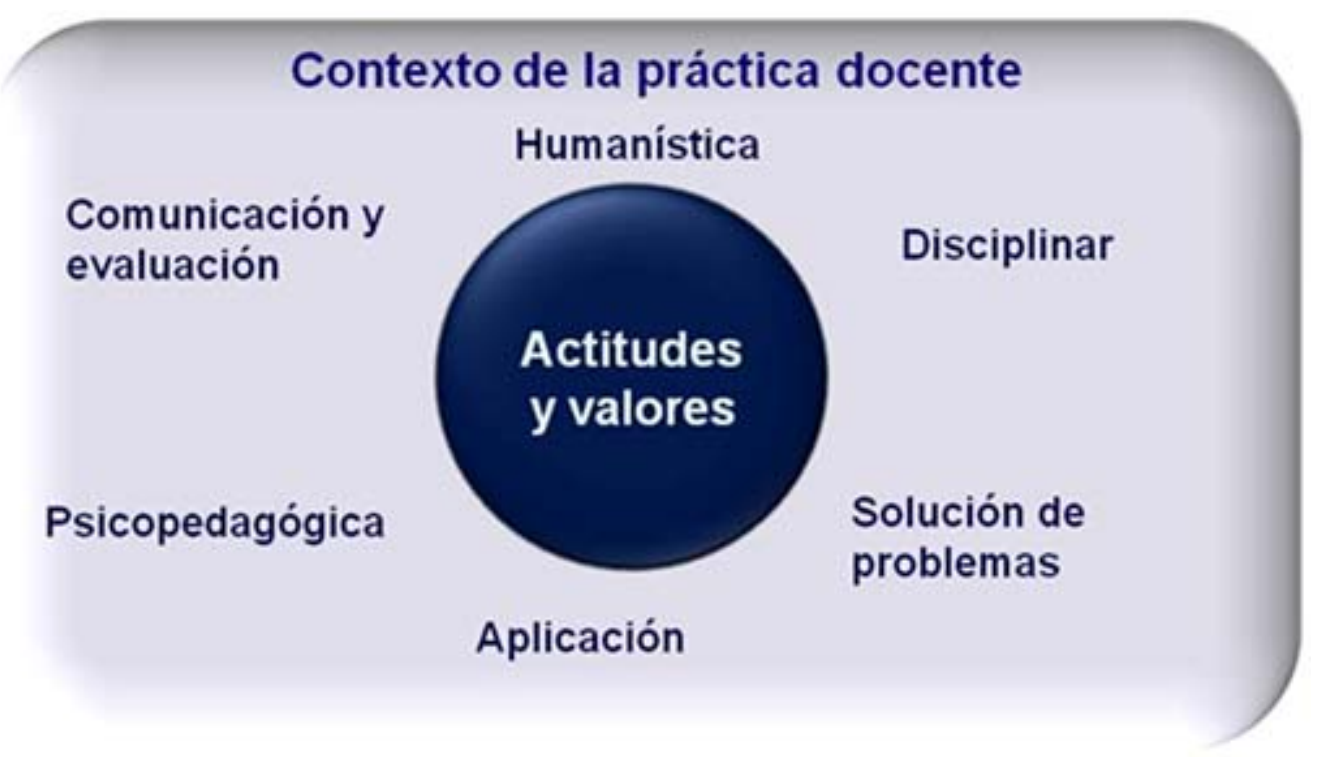

Figura 3. Modelo de Competencias Docentes del Profesor de Medicina

Este nuevo modelo es congruente con la transición al Plan de Estudios de la Facultad de Medicina (2010), donde en términos de evaluación docente se señala que: "Para evaluar el desempeño docente se deben utilizar distintas estrategias complementarias entre las que destacan: evaluación de los docentes mediante la opinión de los estudiantes, por competencias, por logros del aprendizaje de los estudiantes y por autoevaluación, entre otras." Pretendemos promover a corto plazo el desarrollo de otra línea de investigación que implica el uso de los resultados asociados a cada competencia como predictores del desempeño académico de los estudiantes.

De acuerdo con las tendencias actuales de abordaje de la evaluación del desempeño docente, desde una perspectiva multidimensional y multifactorial, de las competencias definidas en el instrumento de autoevaluación (Habilidades docentes y Solución de Problemas), se puede sugerir la propuesta de un modelo más integral, en el cual se fusionen de manera complementaria los procesos de autoevaluación y evaluación basada en la opinión de los estudiantes.

Para la Facultad de Medicina, la evaluación docente debe contribuir a la consecución de las competencias transversales, perfi- les intermedios y de egreso por parte de los estudiantes. Esta debe estar sustentada en la adquisición y aplicación de las competencias adquiridas en su formación básica, sociomédica y clínica, basada en un desempeño satisfactorio de la planta docente, como uno de los pilares centrales en la formación de los estudiantes.

La Figura 4 presenta de manera integrada una visión prospectiva (los elementos marcados en rojo corresponden a la visión del modelo al incorporar la autoevaluación docente), según la cual pudiera orientarse una línea de investigación futura a la clasificación y evaluación de las competencias propuestas en ambos instrumentos, considerando la división de las competencias asociadas en cuatro grandes segmentos (y sustentado lo anterior en los tres grandes bloques de funciones y actividades que involucran la práctica docente en las áreas de formación de los estudiantes y en los perfiles citados):

- La evaluación del estudiante, que brinda una visión única del desarrollo del curso en el momento del proceso educativo.

- La autoevaluación docente puede, en el mismo espacio de clase, ser susceptible de correlacionarse con la evaluación de los estudiantes. 
- La autoevaluación de los aspectos previos a la clase (ligada a la preparación y planeación de clase).
- La autoevaluación ligada al momento posterior a la clase (vinculada con la reflexión y autoevaluación).

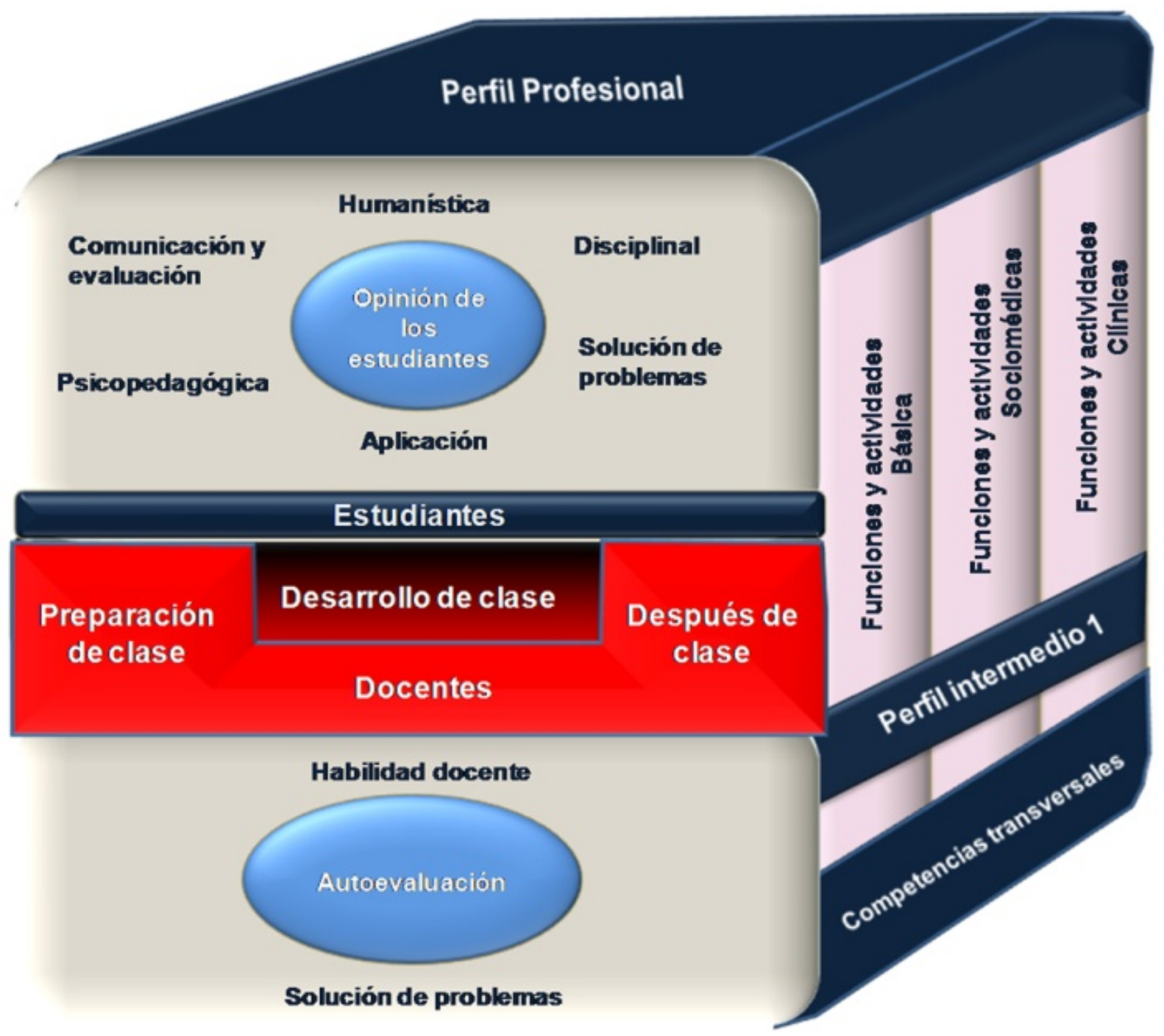

Figura 4. Prospectiva del modelo de competencia docente del profesor de medicina

La presente propuesta resulta un punto de partida sólido para los esfuerzos de la Secretaría de Educación Médica de la Facultad, en el afán de generar un proceso de mejora continua que permita incorporar en el futuro algunas estrategias adicionales a los procesos de evaluación, y en particular de evaluación del desempeño docente, dirigidos a la realimentación y perfeccionamiento de los docentes del ámbito médico.

\section{Referencias}

Asociación Mexicana de Facultades y Escuelas de Medicina (2010). ). Sistema nacional de educación médica. Recuperado el 24 de febrero de 2011 de http://www.amfem.edu.mx

Asociación Nacional de Universidades e Instituciones de Educación Superior (2007). Catálogo de Carreras de Licenciatura en Universidades e Institutos Tecnológicos. Recuperado el 18 de octubre de 2010 de www.anuies.mx 
Castillo, S. (2002). Compromisos de la evaluación educativa. Prentice Hall-España.

Díaz, F. y Rueda, M. (2000). Evaluación de la docencia; perspectivas actuales. México: Paidós

Facultad de Medicina. (2010). Proyecto de modificación del plan de estudios y programas académicos de la licenciatura de Médico Cirujano: 3. Propuesta del plan de estudios. México: Facultad de medicina UNAM.

Fernández, J. (2008). Valoración de la calidad docente: el profesorado. Un modelo de evaluación circular. Madrid: Complutense.

Fluit, C., Bolhuis, S., Grol, R., Laan, R. y Wensing, M. (2010). Assessing the Quality of Clinical Teachers: A Systematic Review of Content and Quality of Questionnaires for Assessing Clinical Teachers. Recuperado el 30 de mayo de 2010 www.ncbi.nlm.nih.gov/pubmed/20703952.

García, B., Loredo, J., Luna, E y Rueda, M. (2008). Modelo de evaluación de competencias docentes para la educación media y superior. En IV Coloquio iberoamericano sobre evaluación de la docencia ( $p$ p 225246). México: UNAM, IISUE. RIED, CONACYT.

Joint Committee (1981). Standards for Educational Evaluation. New York: Mac GrawHill.

Martínez, A., López, J., Herrera, P., Ocampo, J., Petra, I., Uribe, G., García, MC. y Morales, S. (2008). Modelo de competencias del profesor de medicina. a. Educación Médica, 11 (3), 157-167.

Mazón. J., Martínez. J. y Martínez. A. (2009). La evaluación de la función docente mediante la opinión del estudiante. Un nuevo instrumento para nuevas dimensiones: COED. Revista de la Educación Superior, 38 38(1). pp. 113-140.

Montenegro, I. (2003). Evaluación del desempeño docente; fundamentos, modelos $e$ instrumentos. Bogotá: Magisterio.

Nevo, D. (1997). Evaluación basada en el centro; un diálogo para la mejora educativa.va. Bilbao: Mensajero.
Nirenberg, O., Brawerman, J. y Ruiz, V. (2000). Evaluar para la transformación; innovaciones en la evaluación de programas y proyectos sociales. Argentina: Paidós.

Organización para la Cooperación y Desarrollo Económicos (2007). Definition and Selection of Competencies. Recuperado el 28 de septiembre de 2010 http://www.oecd.org/document/17/0,2340, en $2649 \quad 34515 \quad 2669073 \quad 1 \quad 1 \quad 1 \quad 1$, $\underline{00 . h t m l}$

Perrenoud, P. (2007). Diez nuevas competencias para enseñar. México: Graó/Colofón.

Royal College of Physicians and Surgeons of Canada. (2006). The he CANMEDS assessment tools handbook: en introductory guide to assessment methods for the CanMEDS competencies. Canada: CANMEDS.

Rueda, M., y Torquemada, A. (2004). Algunas consideraciones para el diseño de un sistema de evaluación de la docencia en la universidad. En M. Rueda (Ed), ¿Es posible evaluar la docencia en la universidad? Experiencias en México, Canadá, Francia, España y Brasil. (pp. 29-35). México: ANUIES.

Ruiz, J. (1998). Como hacer una evaluación de centros educativos. Madrid: Narcea.

Ruiz, V.M. y Jiménez, J.A. (2004). Estructura de la personalidad: Ortogonalidad versus oblicuidad. Anales de Psicología, vol 20(1), 1-13.

Stufflebeam, D. (1987). Evaluación sistemática: Guía teórica y práctica. Barcelona: Paidós.

Tuning (2010). Tuning América Latina. Recuperado el 9 de noviembre de 2010 de http://tuning.unideusto.org/tuningal/index.p hp?option=content\&task=view\&id=211\&It emid $=240$

Tyler, R. (1977). Principios básicos del currículo. Buenos Aires: Troquel.

Valle. R., Alaminos. I., Contreras. E., Salas. L., Tomasini. P. y Varela. M. (2004). Student Questionnaire to Evaluate Basic Medical Science Teaching (METEBQ-B). Rev. Med. IMSS, 42(5). 
Worthen, B, Sanders, J y Fizpatrick, J. (1997). Program evaluation. Alternative approaches and practical guidelines. New Cork, Addison, Weley: Longman.
Zabala, A. (2002). La práctica educativa: cómo enseñar. Barcelona: Graó.

Zabalza, M. (2007). Competencias docentes del profesor universitario: Calidad y desarrollo profesional. Madrid: Narcea.

\section{ABOUT THE AUTHORS / SOBRE LOS AUTORES}

Flores-Hernández, Fernandoo (fernando.evaluacion@gmail.com). Técnico Académico C TC Asociado, Profesor de pregrado y posgrado de la Facultad de Medicina. Psicometría, Departamento de Evaluación de la Secretaría de Educación Médica. Líneas de trabajo: Evaluación educativa, psicometría, desempeño académico e informática biomédica. Contribución al campo de estudio: Evaluación, metodología de la investigación y análisis multivariados. Su dirección postal es Facultad de Medicina. Secretaría de Educación Médica. Universidad Nacional Autónoma de México. Circuito Interior, Edificio B, 3er. Piso. 04510, Deleg. Coyoacán. Ciudad Universitaria, D.F. Buscar otros artículos de este autor en Scholar Google / Find other articles by this author in Scholar Google

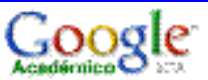

Martínez-González, Adrián (adrianmartinez38@gmail.com). Profesor de Carrera Titular A TC Definitivo, División de Estudios de Posgrado. Jefe del Departamento de Evaluación de la Secretaría de Educación Médica. Líneas de trabajo: Evaluación educativa, metodología de la investigación y ABP. Su dirección postal es Facultad de Medicina. Secretaría de Educación Médica. Universidad Nacional Autónoma de México. Circuito Interior, Edificio B, 3er. Piso. 04510, Deleg. Coyoacán. Ciudad Universitaria, D.F. Buscar otros artículos de este autor en Scholar Google / Find other articles by this author in Scholar Google

\section{Google}

Sánchez-Mendiola, Melchor (melchorsm@gmail.com). Profesor de Carrera Titular A TC Definitivo, División de Estudios de Posgrado. Secretario de Educación Médica de La Facultad de Medicina. Líneas de trabajo: el médico residente como educador, evaluación del aprendizaje, medicina basada en evidencias. Su dirección postal es Facultad de Medicina. Secretaría de Educación Médica. Universidad Nacional Autónoma de México. Circuito Interior, Edificio B, 3er. Piso. 04510, Deleg. Coyoacán. Ciudad Universitaria, D.F. Buscar otros artículos de este autor en Scholar Google / Find other articles by

this author in Scholar Google

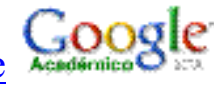

García-Cabrero, Benilde (benilde@servidor.unam.mx). Profesora e investigadora. Líneas de trabajo: desarrollo moral y educación ciudadana, evaluación de la docencia, tecnologías de la información y la comunicación aplicadas a la educación. Contribución al campo de estudio. Evaluación del desempeño docente por competencias. Su dirección postal es: Facultad de Psicología. Universidad Nacional Autónoma de México. Avenida Universidad 3004. Col. Copilco Universidad. Coyoacán, DF CP 04510. Buscar otros artículos de esta autora en Scholar Google / Find other articles by this author in Scholar Google

\section{Google}

Reidl, Lucy M. (lucym@servidor.unam.mx). Profesora Líneas de trabajo: Investigación psicosocial, emociones y salud. Contribución al campo de estudio: Medición, evaluación y metodología de la investigación. Su dirección postal es: Facultad de Psicología. Universidad Nacional Autónoma de México. Avenida Universidad 3004. Col. Copilco Universidad. Coyoacán, DF CP 04510. Buscar otros artículos de esta autora en Scholar Google / Find other articles by this author in Scholar Google

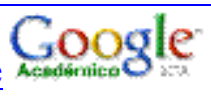


Flores-Hernández, Fernando; Martínez-González, Adrián; Sánchez-Mendiola, Melchor; García-Cabrero, Benilde \& Reidl, Lucy M. (2011). Modelo de competencia docente del profesor de medicina en la UNAM. RELIEVE, v. 17, n. 2, art. 3. http://www.uv.es/RELIEVE/v17n2/RELIEVEv17n2 3.htm

\title{
ARTICLE RECORD / FICHA DEL ARTÍCULO
}

\begin{tabular}{|c|c|}
\hline $\begin{array}{l}\text { Reference / } \\
\text { Referencia }\end{array}$ & 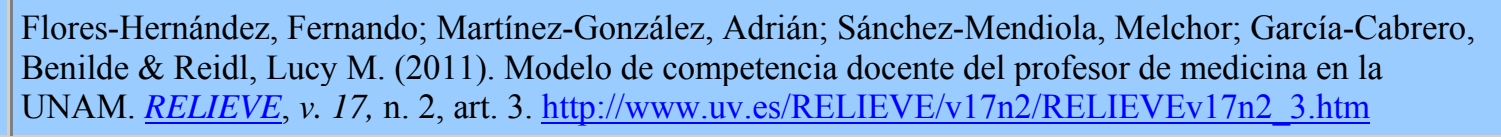 \\
\hline Title / Título & $\begin{array}{l}\text { Modelo de competencia docente del profesor de medicina en la UNAM. [Model of teaching competence } \\
\text { in teachers of medicine at UNAM]. }\end{array}$ \\
\hline $\begin{array}{l}\text { Authors / } \\
\text { Autores }\end{array}$ & $\begin{array}{l}\text { Flores-Hernández, Fernando; Martínez-González, Adrián; Sánchez-Mendiola, Melchor; García-Cabrero, } \\
\text { Benilde \& Reidl, Lucy M. }\end{array}$ \\
\hline $\begin{array}{l}\text { Review / } \\
\text { Revista }\end{array}$ & RELIEVE (Revista ELectrónica de Investigación y EValuación Educativa), v. 17, n. 2 \\
\hline ISSN & $1134-4032$ \\
\hline \begin{tabular}{l|} 
Publication date \\
Fecha de \\
publicación
\end{tabular} & $\begin{array}{l}2011 \text { (Reception Date: } 2011 \text { Juny } 3 \text {; Approval Date: } 2011 \text { September 22. Publication Date: } 2011 \text { Sep- } \\
\text { tember 26). }\end{array}$ \\
\hline $\begin{array}{l}\text { Abstract / } \\
\text { Resumen }\end{array}$ & $\begin{array}{l}\text { The study presents a model of teaching competence in teachers of medicine. It was generated from an } \\
\text { existing conceptual model: a) it was referred to the opinion of experts who selected the competencies of } \\
\text { greater impact; b) a reference profile was constructed; c) an instrument was generated based on student } \\
\text { opinion, and another for self-assessment; d) the instruments were validated by } 18 \text { experts in evaluation; } \\
\text { e) they were applied to 2,281 students and } 107 \text { teachers, obtaining adequate psychometric characteris- } \\
\text { tics, establishing a model according to current trends in teacher performance assessment. } \\
\text { El estudio presenta un modelo de competencia docente del profesor de medicina. Fue generado a partir de } \\
\text { un modelo conceptual preexistente: a) dicho modelo se sometió al juicio de expertos quienes selecciona- } \\
\text { ron las competencias de mayor impacto; b) se construyó un perfil de competencias; c) se generó un ins- } \\
\text { trumento basado en la opinión de los estudiantes y otro de autoevaluación; d) ambos fueron validados por } \\
18 \text { expertos en evaluación; e) se aplicó a } 2,281 \text { estudiantes y } 107 \text { profesores, obteniendo un comporta- } \\
\text { miento psicométrico adecuado que permitió establecer un modelo acorde a las tendencias actuales en } \\
\text { evaluación del desempeño docente. }\end{array}$ \\
\hline $\begin{array}{l}\text { Keywords / } \\
\text { Descriptores }\end{array}$ & $\begin{array}{l}\text { Teachers, Assessment, Competence, Model, Performance. } \\
\text { Docencia, Evaluación, Competencias, Modelo, Desempeño }\end{array}$ \\
\hline $\begin{array}{l}\text { Institution / Ins- } \\
\text { titución }\end{array}$ & Facultad de Medicina. Universidad Nacional Autónoma de Mexico. \\
\hline $\begin{array}{l}\text { Publication site / } \\
\text { Dirección }\end{array}$ & http://www.uv.es/RELIEVE \\
\hline $\begin{array}{l}\text { Language / } \\
\text { Idioma }\end{array}$ & Español (Title, abstract and keywords in English \& Spanish) \\
\hline
\end{tabular}

\section{RELIEVE}

\section{Revista ELectrónica de Investigación y $\mathbf{E V}$ aluación Educativa E-Journal of Educational Research, Assessment and Evaluation}

[ISSN: 1134-4032]

\begin{abstract}
(C) Copyright, RELIEVE. Reproduction and distribution of this articles it is authorized if the content is no modified and their origin is indicated (RELIEVE Journal, volume, number and electronic address of the document).

(C) Copyright, RELIEVE. Se autoriza la reproducción y distribución de este artículo siempre que no se modifique el contenido y se indique su origen (RELIEVE, volumen, número y dirección electrónica del documento).
\end{abstract}

